Article

\title{
Neutrophil-to-Lymphocyte Ratio Is a Predictive Biomarker in Patients with Epidermal Growth Factor Receptor (EGFR) Mutated Advanced Non-Small Cell Lung Cancer (NSCLC) Treated with Tyrosine Kinase Inhibitor (TKI) Therapy
}

\author{
Nicole K. Yun ${ }^{1}{ }^{\mathbb{D}}$, Sherin J. Rouhani ${ }^{2}$, Christine M. Bestvina ${ }^{2}$, Ethan M. Ritz ${ }^{3}$, Brendan A. Gilmore ${ }^{4}$, \\ Imad Tarhoni ${ }^{5}$, Jeffrey A. Borgia ${ }^{5}$, Marta Batus ${ }^{4}$, Philip D. Bonomi ${ }^{4}$ and Mary Jo Fidler ${ }^{4, *}$ \\ 1 Department of Internal Medicine, Rush University Medical Center, Chicago, IL 60612, USA; \\ nicole_k_yun@rush.edu \\ 2 Section of Hematology/Oncology, Department of Medicine, The University of Chicago Comprehensive \\ Cancer Center, Chicago, IL 60637, USA; Sherin.rouhani@uchospitals.edu (S.J.R.); \\ cbestvina@medicine.bsd.uchicago.edu (C.M.B.) \\ 3 Bioinformatics and Biostatistics Core, Rush University Medical Center, Chicago, IL 60612, USA; \\ Ethan_M_Ritz@rush.edu \\ 4 Hematology, Oncology and Cell Therapy, Department of Medicine, Rush University Medical Center, \\ Chicago, IL 60612, USA; Brendan_gilmore@rush.edu (B.A.G.); Marta_Batus@rush.edu (M.B.); \\ Philip_Bonomi@Rush.edu (P.D.B.) \\ Citation: Yun, N.K.; Rouhani, S.J.; \\ Bestvina, C.M.; Ritz, E.M.; Gilmore, \\ B.A.; Tarhoni, I.; Borgia, J.A.; Batus, \\ Cell \& Molecular Medicine, Pathology, Rush University Medical Center, Chicago, IL 60612, USA; \\ Imad_Tarhoni@rush.edu (I.T.); Jeffrey_A_Borgia@rush.edu (J.A.B.) \\ * Correspondence: Mary_Fidler@rush.edu
} M.; Bonomi, P.D.; Fidler, M.J. Neutrophil-to-Lymphocyte Ratio Is a Predictive Biomarker in Patients with Epidermal Growth Factor Receptor (EGFR) Mutated Advanced Non-Small Cell Lung Cancer (NSCLC) Treated with Tyrosine Kinase Inhibitor (TKI) Therapy. Cancers 2021, 13, 1426. https:// doi.org/10.3390/cancers13061426

Academic Editor: Federico Cappuzzo

Received: 24 January 2021

Accepted: 16 March 2021

Published: 20 March 2021

Publisher's Note: MDPI stays neutral with regard to jurisdictional claims in published maps and institutional affiliations.

Simple Summary: Though patients with sensitizing epidermal growth factor receptor (EGFR) mutated non-small cell lung cancer have been known to have rapid and durable responses to tyrosine kinase inhibitors, a subset of patients have inferior outcomes and may benefit from therapy escalation. Neutrophil-to-lymphocyte ratio (NLR) has been recognized as one indicator of poor prognosis in patients with various cancers, including non-small cell lung cancer. It is associated with cancer cachexia and treatment resistance. The aim of our study is to assess the serial predictive value of NLR and other markers of cachexia and chronic inflammation in the subset of patients with advanced non-small cell lung cancer who harbor the epidermal growth factor receptor mutation and who are undergoing treatment with tyrosine kinase inhibitor therapy.

Abstract: Background: First-line treatment for patients with non-small cell lung cancer (NSCLC) with a sensitizing epidermal growth factor receptor (EGFR) mutation is a tyrosine kinase inhibitor (TKI). Despite higher response rates and prolonged progression free survival (PFS) compared with platinum doublet chemotherapy, a subset of these patients do not receive prolonged benefit from these agents. We investigate if the neutrophil-to-lymphocyte ratio (NLR) and other markers of cachexia and chronic inflammation correlate with worse outcomes in these patients. Methods: This study is a retrospective review of 137 patients with advanced EGFR-mutated NSCLC treated with TKIs at Rush University Medical Center and University of Chicago Medicine from August 2011 to July 2019, with outcomes followed through July 2020. The predictive value of NLR and body mass index (BMI) was assessed at the start of therapy, and after 6 and 12 weeks of treatment by univariable and multivariable analyses. Results: On univariable analysis, NLR $\geq 5$ or higher NLR on a continuous scale were both associated with significantly worse PFS and overall survival (OS) at treatment initiation, and after 6 or 12 weeks of treatment. On multivariable analysis, NLR $\geq 5$ was associated with increased risk of death at 12 weeks of therapy (HR 3.002, 95\% CI 1.282-7.029, $p=0.011$ ), as was higher NLR on a continuous scale (HR 1.231, 95\% CI 1.063-1.425, $p=0.0054$ ). There was no difference in PFS and OS and amongst BMI categories though number of disease sites and Eastern Cooperative Oncology Group (ECOG) performance status was associated with worse PFS and OS. Conclusions: Patients with NLR $\geq 5$ have a worse median PFS and median OS than patients with NLR $<5$. NLR may have value as a predictive 
biomarker and may be useful for selecting patients for therapy intensification in the front-line setting either at diagnosis or after 12 weeks on therapy. NLR needs to be validated prospectively.

Keywords: tyrosine kinase inhibitor (TKI); neutrophil-to-lymphocyte ratio (NLR); predictive biomarker; body mass index (BMI); epidermal growth factor receptor (EGFR); advanced non-small cell lung cancer (NSCLC)

\section{Introduction}

Lung cancer remains the leading cause of cancer death among both men and women worldwide [1]. Lung cancer patients with sensitizing driver mutations in the epidermal growth factor receptor (EGFR) gene make up approximately $15 \%$ of the NSCLC patient overall. In recent years, the prognosis and quality of life of patients with advanced NSCLC harboring EGFR mutations has changed dramatically due to the incorporation of tyrosine kinase inhibitors (TKIs), now standard of care as first-line therapy [2]. Median overall survival (mOS) has increased to 38.6 months with osimertinib with the additional benefit of a more tolerable side effect profile compared with prior generation TKI therapy or cytotoxic chemotherapy [3]. While there is a group of patients with exon 20 insertions and other uncommon EGFR mutations that are not sensitive to the current generations of TKIs, there is also a group of patients with known sensitizing mutations who, nonetheless, exhibit primary resistance and can progress in as little as 3 months [4].

Cancer-associated inflammation in the tumor microenvironment and systemic inflammation can lead to tumor growth and therapy resistance. Myeloid-derived suppressor cells (MDSCs) and tumor associated neutrophils (TAN) can inhibit the immune response through a variety of direct and soluble mechanisms. Lymphocytes consist of $B$ cells which produce antibodies, and T cells which help activate B cells and which also have direct cytotoxic activity against tumor cells. Therefore, the immune response profile can serve as a useful tool to predict patient prognosis and response to treatment. The neutrophil-to-lymphocyte ratio (NLR) and platelet-to-lymphocyte ratios (PLR) have both been investigated as potential prognostic and predictive biomarkers that are convenient and easy to obtain with routine lab tests. An elevated NLR has emerged as an indicator of poor prognosis across a variety of tumor types [5-8]. Wang et al conducted a 2019 meta-analysis looking at pretreatment NLR for NSCLC patients receiving systemic therapy including chemotherapy, immunotherapy and targeted therapy. The authors reported that an elevated pretreatment NLR is consistently associated with inferior survival [9].

More recently, reports have begun to comment on NLR as a biomarker for predicting prognosis and response to treatment in patients with advanced, EGFR-mutated NSCLC who are treated with TKI therapy $[2,8,10,11]$. Most of the studies investigating NLR in EGFR-mutated NSCLC have been conducted in predominantly Asian populations [8,10-13]. Although there have also been similar reports in European [14] and Australian populations [15], there is little information in an African American population. Prior studies looking at patients with EGFR-mutated NSCLC undergoing treatment with TKIs have used variable NLR cutoff values, frequently chosen to reflect the top quartile of the study population or to maximize the receiver operating characteristic (ROC) curve. These cutoffs generally range from $3-5$, but an optimal value has not been validated. In concordance with several prior studies [5-15], we have elected to use an NLR cutoff of 5 to provide greater discriminatory potential. Additionally, there have been few studies to date including patients treated with osimertinib, a third generation TKI which was FDA approved in 2017. The purpose of our study is to validate the NLR as a predictive indicator at different time points during therapy, and to examine other factors potentially contributing to heightened systemic inflammatory response such as baseline number of metastatic sites and therapyrelated toxicities. Since cancer cachexia can be an indicator of a pro-inflammatory state and $\mathrm{BMI}<18.5$ has also been reported as a poor prognostic marker [10], we also investigate the 
relationship between changes in BMI with changes in NLR, progression free survival (PFS), and overall survival (OS).

\section{Materials and Methods}

\subsection{Patient Selection and Data Collection}

We retrospectively analyzed 137 patients with advanced non-small cell lung cancer treated at Rush University Medical Center or University of Chicago Medicine from August 2011 to July 2019. Outcomes were followed through 1 July 2020. Patients aged 18 years or older with advanced non-small cell lung cancer and a targetable EGFR mutation, including exon 19 deletions, L858R, L861Q, S768I, G709A, and G719S mutations, were included. EGFR mutations with known TKI resistance such as exon 20 insertions were excluded. All patients received oral EGFR tyrosine kinase inhibitor therapy (erlotinib, gefitinib, afatinib, osimertinib), and progression free survival and overall survival were measured from time of first TKI treatment.

For each patient, the NLR was recorded at the time of TKI initiation, 6 weeks after starting TKI and 12 weeks after starting TKI. The values were taken from routine complete blood count $(\mathrm{CBC})$ with differential drawn closest to the respective time point \pm 30 days. Most CBC were drawn at outpatient appointments. Treatment responses were evaluated using computed tomography (CT) every 8-12 weeks per routine clinical care. For those who experienced progression while on TKI, we recorded the NLR at the time of progression and at 6 weeks prior to progression. Body mass index (BMI) of the patient was also collected at the time of diagnosis, time of therapy initiation, 6 weeks after therapy, 12 weeks after therapy, and at progression and 6 weeks prior, if applicable.

We defined PFS as time from start of TKI to time of disease progression on imaging, such as interval increase in size of primary or metastatic lesion, increase in size of malignant pleural effusion, or new metastatic disease that resulted in a clinical change in therapy. Due to the number of patients having nonmeasurable disease and nontarget lesions, we were unable to use Response Evaluation Criteria in Solid Tumors (RECIST) to define tumor progression. In cases of oligoprogression treated with local ablative therapy, disease was considered progressive only if the scan results triggered a change in systemic therapy, including switching to a third generation TKI or chemotherapy. OS was calculated as time from start of TKI until time of death or last clinical follow-up.

Drug toxicities attributed to TKI therapy were graded by severity in accordance with version 5 of the Common Terminology Criteria for Adverse Events (CTCAE). We recorded the highest grade experienced by the patient throughout the entire duration of treatment with TKI.

Baseline number of disease sites was used to quantify disease burden and account for varying degrees of tumor involvement between patients. By referring to initial staging $C T$, positron emission tomography (PET) scan and/or magnetic resonance imaging (MRI) at time of diagnosis, the following sites of disease were recorded: lung, regional lymph nodes (LN), distant LN, pleura, heart/pericardium, bone, liver, adrenal, brain, and other. The distinction between regional and distant LN were made based on the TNM staging system.

\subsection{Statistics}

PFS and OS were computed using the Kaplan-Meier method and compared using the log rank test. Spearman rho $(\rho)$ was used to correlate number of disease sites with NLR at diagnosis. T-tests were used to compare mean NLR between patients with any high grade $(\geq 3)$ toxicity versus those with no or low (grades $0-2$ ) toxicity. To assess whether or not a relationship existed between NLR and BMI throughout the course of TKI therapy, changes in NLR and BMI were calculated by subtracting the value at treatment start from the values at 6 or 12 weeks, and the relationship between the change in NLR vs. BMI was analyzed using Pearson correlation. Cox regression was used to assess correlation between change in NLR and BMI with PFS and OS at 6 weeks and 12 weeks. Univariable and multivariable analysis was also used to compare PFS and OS in patients with 
varying baseline characteristics: age at diagnosis, gender, race, smoking status, BMI, total number of metastatic sites, central nervous system (CNS) metastases, first-line TKI, other systemic treatment prior to TKI initiation, Eastern Cooperative Oncology Group (ECOG) performance status, toxicity grade, and mutation. Multivariable cox regression analysis was performed to assess the independent predictive value of NLR as well as significance of other baseline characteristics. All analyses were conducted using $\mathrm{R}$ version 4.0.3.

\section{Results}

\subsection{Patient Characteristics}

In total, 137 patients treated at Rush University Medical Center or University of Chicago Medicine from August 2011 to July 2019 were retrospectively analyzed. Baseline demographics with classification criteria are shown in Table 1. Median age at diagnosis was 63.9 years, with an interquartile range of 56.7 to 71.9 years. Our patient population was predominantly female (102/137 patients, $74.4 \%)$, and $45.2 \%$ were never smokers. Patient race was $62.8 \%$ white, $21.9 \%$ black or African American, and $8.8 \%$ Asian. A total of $51.1 \%$ of patients had an exon 19 deletion, 33.3\% had L858R, and 5.0\% had T790M on their initial genetic testing.

Table 1. Baseline patient characteristics and distribution divided by neutrophil-to-lymphocyte ratio (NLR) at therapy start.

\begin{tabular}{|c|c|c|c|c|}
\hline Patient Characteristics & $\mathrm{NLR}^{1}<5$ & NLR $\geq 5$ & NLR N/A ${ }^{2}$ & Total \\
\hline \multicolumn{5}{|l|}{ Age at Diagnosis } \\
\hline$<65$ & 46 & 23 & 4 & 73 \\
\hline$\geq 65$ & 42 & 17 & 5 & 64 \\
\hline \multicolumn{5}{|l|}{ Gender } \\
\hline Male & 19 & 14 & 2 & 35 \\
\hline Female & 69 & 26 & 7 & 102 \\
\hline \multicolumn{5}{|l|}{ Race } \\
\hline White & 53 & 26 & 7 & 86 \\
\hline Asian & 9 & 3 & 0 & 12 \\
\hline Black/African American & 20 & 8 & 2 & 30 \\
\hline Other & 4 & 1 & 0 & 5 \\
\hline More than one & 1 & 0 & 0 & 1 \\
\hline Not reported & 1 & 2 & 0 & 3 \\
\hline \multicolumn{5}{|l|}{ Smoking Status } \\
\hline Never & 41 & 17 & 4 & 62 \\
\hline$\leq 15$ pack years & 30 & 12 & 2 & 44 \\
\hline$>15$ pack years & 17 & 10 & 3 & 30 \\
\hline Former (not counted) & 0 & 1 & 0 & 1 \\
\hline \multicolumn{5}{|l|}{ Baseline $\mathrm{BMI}^{3}$} \\
\hline$\leq 18.5$ & 0 & 2 & 0 & 2 \\
\hline$>18.5$ to $\leq 25$ & 36 & 23 & 3 & 62 \\
\hline$>25$ to $\leq 30$ & 31 & 10 & 3 & 44 \\
\hline$>30$ & 20 & 5 & 3 & 28 \\
\hline Not available & 1 & 0 & 0 & 1 \\
\hline \multicolumn{5}{|l|}{ Number of Metastatic Sites } \\
\hline 1 & 8 & 1 & 1 & 10 \\
\hline 2 & 29 & 1 & 3 & 33 \\
\hline 3 & 25 & 14 & 3 & 42 \\
\hline 4 & 16 & 11 & 1 & 28 \\
\hline 5 & 8 & 8 & 0 & 16 \\
\hline 6 & 0 & 1 & 0 & 1 \\
\hline 7 & 2 & 4 & 1 & 7 \\
\hline \multicolumn{5}{|l|}{$\mathrm{CNS}^{4}$ Metastases } \\
\hline Present & 25 & 21 & 1 & 47 \\
\hline Not present & 63 & 19 & 8 & 90 \\
\hline
\end{tabular}


Table 1. Cont.

\begin{tabular}{|c|c|c|c|c|}
\hline Patient Characteristics & $\operatorname{NLR}^{1}<5$ & NLR $\geq 5$ & NLR N/A ${ }^{2}$ & Total \\
\hline \multicolumn{5}{|l|}{ Histology } \\
\hline Adenocarcinoma & 86 & 40 & 9 & 135 \\
\hline Mixed/poorly differentiated NSCLC ${ }^{5}$ & 2 & 0 & 0 & 2 \\
\hline \multicolumn{5}{|l|}{ Baseline Mutations } \\
\hline Exon 19 Deletion (Total with All Co-mutations) & 49 & 21 & 2 & 72 \\
\hline Exon 19 alone & 46 & 21 & 2 & 69 \\
\hline$+\mathrm{T} 790 \mathrm{M}$ & 2 & 0 & 0 & 2 \\
\hline$+E G F R^{6}$ amplification & 1 & 0 & 0 & 1 \\
\hline$+\mathrm{L} 858 \mathrm{R}$ & 1 & 0 & 0 & 1 \\
\hline L858R (Total with All Co-mutations) & 30 & 15 & 2 & 47 \\
\hline L858R alone & 24 & 14 & 2 & 40 \\
\hline +T790M & 4 & 1 & 0 & 5 \\
\hline +Exon 19 & 1 & 0 & 0 & 1 \\
\hline$+\mathrm{L} 833 \mathrm{~V}$ & 1 & 0 & 0 & 1 \\
\hline T790M (Total with All Co-mutations) & 6 & 1 & 0 & 7 \\
\hline+ Exon 19 & 2 & 0 & 0 & 2 \\
\hline$+\mathrm{L} 858 \mathrm{R}$ & 4 & 1 & 0 & 5 \\
\hline G719X (Total with All Co-mutations) & 6 & 3 & 0 & 9 \\
\hline G719A alone & 1 & 1 & 0 & 2 \\
\hline G719A + L833_V834delinsFL & 1 & 0 & 0 & 1 \\
\hline $\mathrm{G} 719 \mathrm{~A}+\mathrm{S} 768 \mathrm{I}$ & 1 & 0 & 0 & 1 \\
\hline G719C + S768I & 1 & 0 & 0 & 1 \\
\hline G719A + E709A & 1 & 0 & 0 & 1 \\
\hline G719S + G709A & 1 & 0 & 0 & 1 \\
\hline $\mathrm{G} 719 \mathrm{~S}+\mathrm{E} 709 \mathrm{~A}$ & 0 & 2 & 0 & 2 \\
\hline S768I (Total with All Co-mutations) & 3 & 0 & 1 & 4 \\
\hline S768I alone & 1 & 0 & 1 & 2 \\
\hline S768I +G719X & 2 & 0 & 0 & 2 \\
\hline L861Q Alone & 1 & 0 & 1 & 2 \\
\hline \multicolumn{5}{|l|}{ Others } \\
\hline Exon 18 del $p . E 709 \_T 710 d e l i n s D$ & 0 & 0 & 1 & 1 \\
\hline LUL: Exon 19; RUL: pT790M and L861Q & 0 & 0 & 1 & 1 \\
\hline EGFR amplification and E709_T710 > D; & 0 & 0 & 1 & 1 \\
\hline Not known & 1 & 1 & 0 & 2 \\
\hline \multicolumn{5}{|l|}{ First-line TKI ${ }^{7}$} \\
\hline Erlotinib & 48 & 21 & 7 & 76 \\
\hline Osimertinib & 26 & 9 & 1 & 36 \\
\hline Afatinib & 13 & 10 & 1 & 24 \\
\hline Gefitinib & 1 & 0 & 0 & 1 \\
\hline \multicolumn{5}{|l|}{ TKI Began 1+ Years after Advanced Stage Diagnosis } \\
\hline Yes & 7 & 3 & 0 & 10 \\
\hline No & 81 & 37 & 9 & 127 \\
\hline \multicolumn{5}{|c|}{ Other Systemic Treatment for Metastatic Disease before Starting TKI } \\
\hline Yes & 28 & 8 & 0 & 36 \\
\hline No & 60 & 32 & 9 & 101 \\
\hline \multicolumn{5}{|l|}{ ECOG $^{8}$ Score } \\
\hline 0 & 30 & 9 & 5 & 44 \\
\hline 1 & 49 & 23 & 4 & 76 \\
\hline 2 & 6 & 6 & 0 & 12 \\
\hline 3 & 2 & 2 & 0 & 4 \\
\hline Not reported & 1 & 0 & 0 & 1 \\
\hline \multicolumn{5}{|l|}{ Toxicity Grade } \\
\hline Grade 0-2 & 62 & 31 & 5 & 98 \\
\hline Grade 3-4 & 25 & 8 & 4 & 37 \\
\hline Grade 5 & 1 & 1 & 0 & 2 \\
\hline
\end{tabular}

1. NLR, neutrophil-to-lymphocyte ratio; 2. BMI, body mass index; 3. N/A, not available; 4. CNS, central nervous system; 5. NSCLC, non-small cell lung cancer; 6. EGFR, epidermal growth factor receptor; 7. TKI, tyrosine kinase inhibitor; 8. ECOG, Eastern Cooperative Oncology Group. 
The majority (54.7\%) of patients received first-line treatment with erlotinib (Table 1). Importantly, $26 \%$ of patients received first-line treatment with osimertinib. The proportion of patients on each therapy with an NLR $<5$ compared to NLR $\geq 5$ was relatively similar at the time of therapy initiation (Table 2).

Table 2. Distribution of TKI use at time of therapy start, 6 weeks and 12 weeks for patients with NLR $<5$ and NLR $\geq 5$.

\begin{tabular}{ccccccc}
\hline TKI & \multicolumn{2}{c}{ Therapy Start } & \multicolumn{2}{c}{$\mathbf{6}$ Weeks } & \multicolumn{2}{c}{$\mathbf{1 2}$ Weeks } \\
\hline Drug & NLR $<5$ & NLR $\geq \mathbf{5}$ & NLR $<\mathbf{5}$ & NLR $\geq \mathbf{5}$ & NLR $<5$ & NLR $\geq \mathbf{5}$ \\
\hline Afatinib & $15 \%$ & $15 \%$ & $19 \%$ & $12 \%$ & $19 \%$ & $15 \%$ \\
Erlotinib & $54.50 \%$ & $32 \%$ & $50 \%$ & $69 \%$ & $59 \%$ & $50 \%$ \\
Gefitinib & $1 \%$ & $0 \%$ & $1 \%$ & $0 \%$ & $1 \%$ & $0 \%$ \\
Osimertinib & $29.50 \%$ & $53 \%$ & $30 \%$ & $19 \%$ & $21 \%$ & $35 \%$ \\
\hline
\end{tabular}

\subsection{Univariable Analysis on NLR and Survival Outcomes}

Median follow-up time from date of TKI initiation was 36.6 months, with a maximum of 103.4 months and a minimum of 6.3 months. The PFS and OS curves are depicted in Figure 1 with results summarized in Table 3 . At therapy start, patients with NLR $<5(n=88)$ had a median progression free survival (mPFS) of 17.2 months and a mOS of 58.0 months, while those with NLR $\geq 5(n=40)$ had a mPFS of 14.0 months $(p=0.0029)$ and mOS of 27.6 months $(p=0.0024)$. After 6 weeks of treatment, patients with NLR $<5(n=104)$ had a mPFS of 17.4 months and mOS of 59.7 months, while those with NLR $\geq 5(n=27)$ had a mPFS of 12.1 months $(p=0.0037)$ and a mOS of 39.1 months $(p=0.015)$. After 12 weeks of treatment, patients with NLR $<5(n=89)$ had a mPFS of 18.4 months and mOS of 59.7 months, while those with NLR $\geq 5(n=21)$ had a mPFS of 5.8 months $(p=0.0003)$ and a mOS of 25.2 months

$(p=0.00083)$.

At time of progression, patients with NLR $<5(n=71)$ had a mOS of 33.4 months, while those with NLR $\geq 5$ had a mOS of 10.2 months $(p=0.00022)$. Survival outcomes were also analyzed using NLR $\geq 3$ and NLR $<3$ to assess its predictive value at another commonly used threshold. A similar association was seen where NLR $\geq 3$ predicted worse OS at treatment initiation and after 12 weeks; results are summarized in Figure S1 and Table S1.

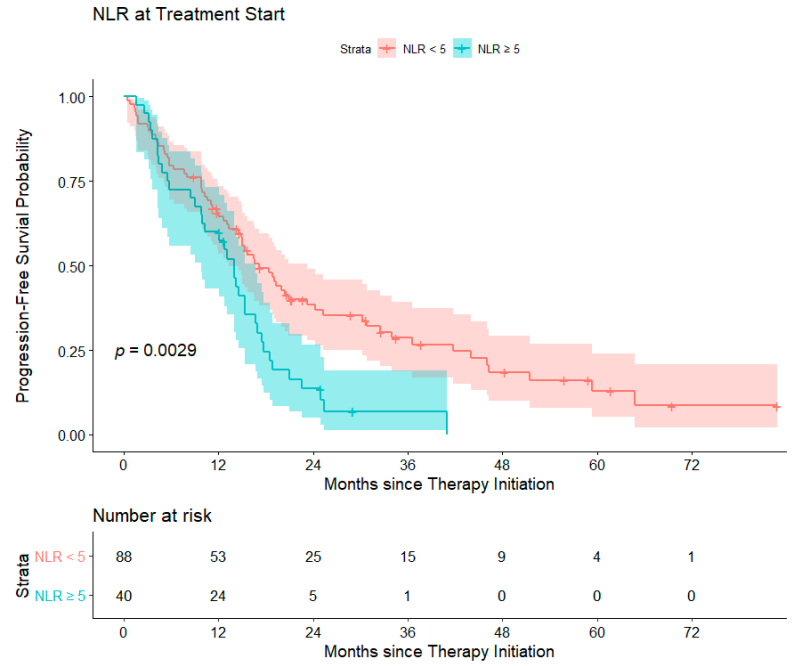

(a)

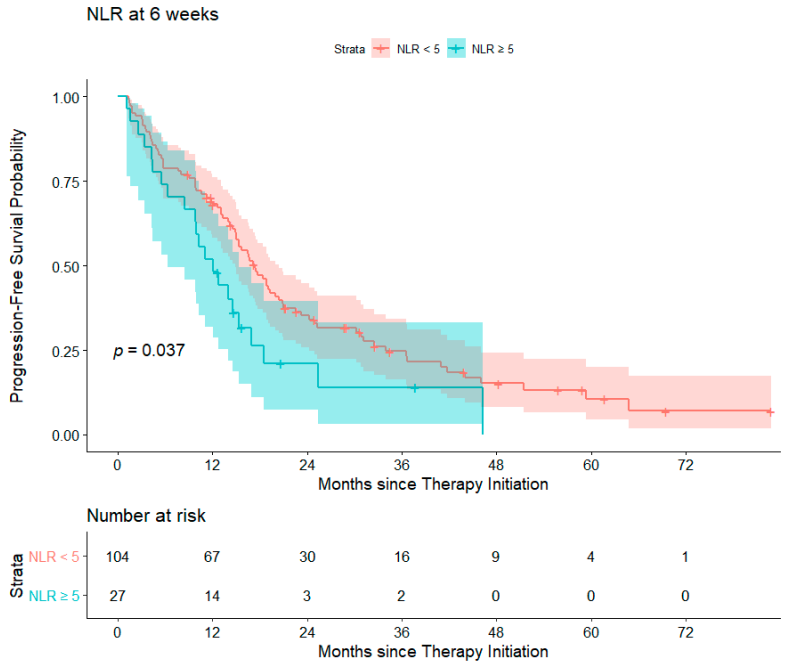

(b)

Figure 1. Cont. 

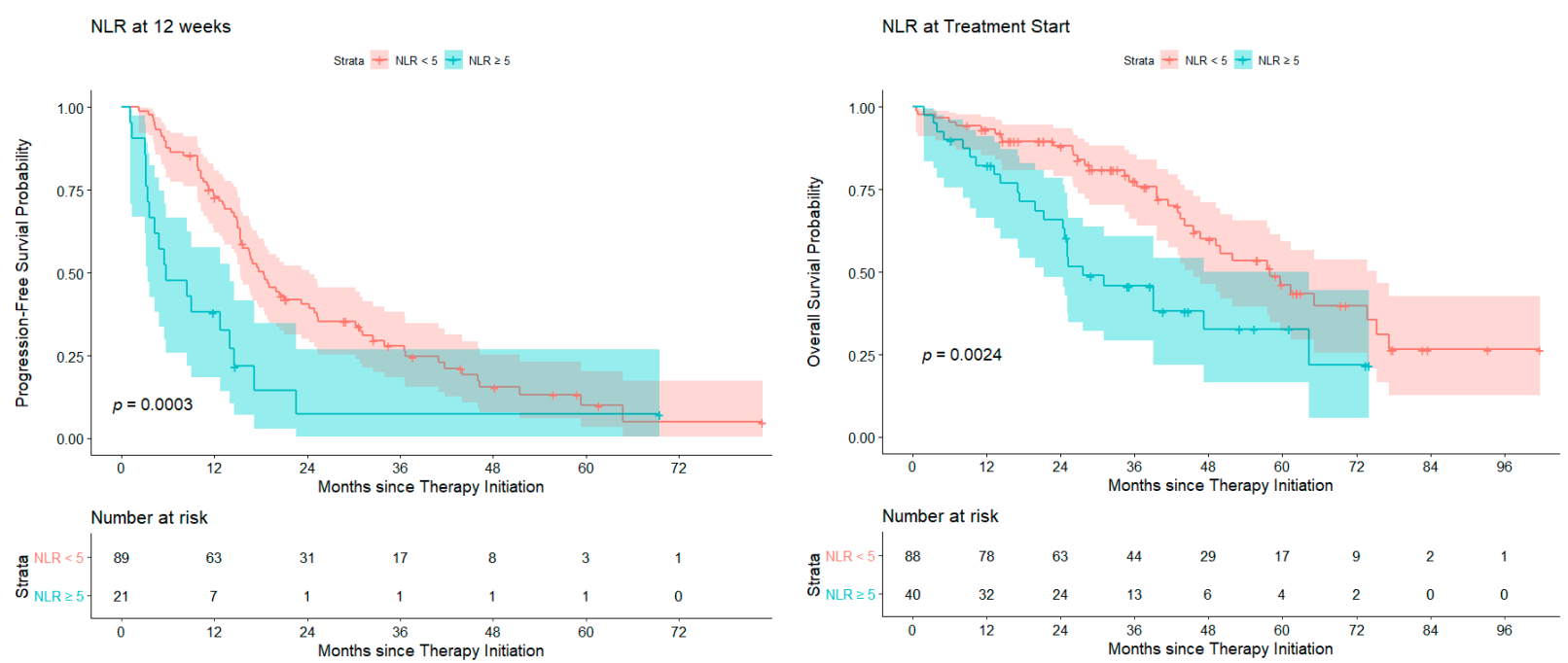

(c)

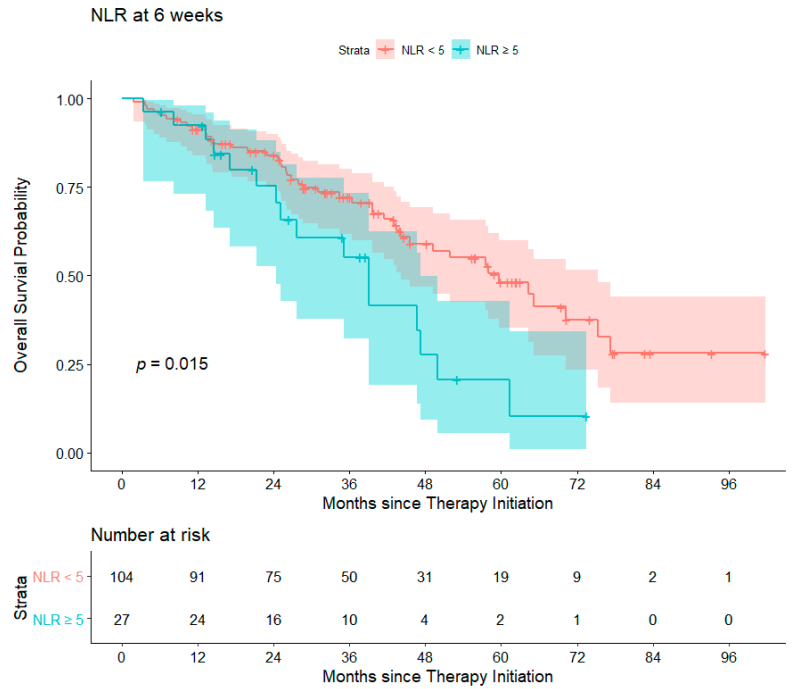

(e) (d)

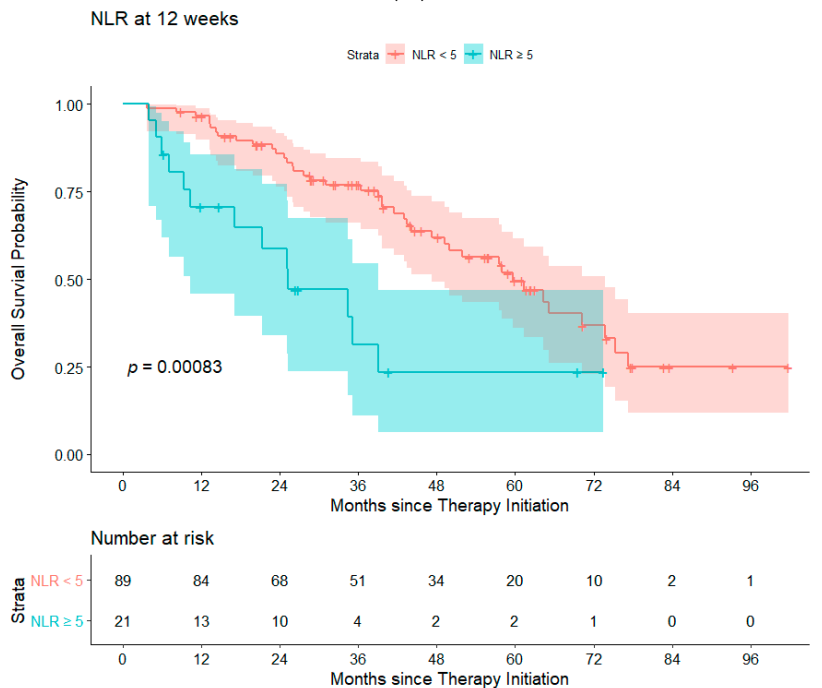

(f)

Figure 1. Progression free survival probability for NLR $<5$ (blue) compared to NLR $\geq 5$ (red) at (a) treatment start, (b) 6 weeks of therapy and (c) 12 weeks of therapy. Overall survival probability for NLR $<5$ compared to NLR $\geq 5$ at (d) treatment start, (e) 6 weeks of therapy and (f) 12 weeks of therapy. Abbreviations: NLR, neutrophil-to-lymphocyte ratio.

When assessing NLR as a continuous variable without adjusting for covariates, there was an increased risk for progression at treatment start (hazard ratio (HR) 1.050, 95\% confidence interval (CI) 1.010-1.092, $p=0.0137$ ), at 6 weeks (HR 1.073, 95\% CI 1.022-1.127, $p=0.0047$ ) and at 12 weeks (HR 1.169, 95\% CI 1.080-1.266, $p=0.0001$ ). There was also an increased risk for death at treatment start (HR 1.060, 95\% CI 1.013-1.109, $p=0.0113$ ), 6 weeks (HR 1.087, 95\% CI 1.027-1.152, $p=0.0043$ ) and at 12 weeks (HR 1.181, 95\% CI $1.063-1.312, p=0.002)$

Patients who had a decrease in NLR did not have significantly better PFS or OS compared to patients whose NLR increased at either 6 weeks $(p=0.29)$ or 12 weeks $(p=0.52)$. Furthermore, there was no significant change in the NLR from the start of treatment to 6 weeks prior to progression (mean $=-0.9702$, standard deviation $(\mathrm{SD})=5.6207, n=86$, $p=0.1786$ ), or to the time of progression (mean $=0.4406, \mathrm{SD}=7.3476, n=96, p=0.5582$ ). 
Table 3. Progression free survival (PFS) and overall survival (OS) for patients with NLR $\geq 5$ and $<5$ at therapy start, 6 weeks and 12 weeks after beginning treatment.

\begin{tabular}{|c|c|c|c|c|c|c|}
\hline Outcome & NLR & $\begin{array}{l}\text { Measurement } \\
\text { Time }\end{array}$ & $n$ & $\begin{array}{l}\text { Median } \\
\text { (Months) }\end{array}$ & $95 \%$ CI & $p$-Value \\
\hline OS & $<5$ & Treatment Start & 88 & 58 & $(45.5,75.3)$ & \multirow{2}{*}{0.0024} \\
\hline OS & $\geq 5$ & Treatment Start & 40 & 27.6 & $(21.3,47.3)$ & \\
\hline OS & $<5$ & $\begin{array}{l}6 \text { weeks after } \\
\text { Treatment Start }\end{array}$ & 104 & 59.7 & $(44.2,75.3)$ & \multirow{2}{*}{0.015} \\
\hline OS & $\geq 5$ & $\begin{array}{l}6 \text { weeks after } \\
\text { Treatment Start }\end{array}$ & 27 & 39.1 & $(24.3,47.3)$ & \\
\hline OS & $<5$ & $\begin{array}{l}12 \text { weeks after } \\
\text { Treatment Start }\end{array}$ & 89 & 59.7 & $(47.3,73.8)$ & \multirow{2}{*}{0.00083} \\
\hline OS & $\geq 5$ & $\begin{array}{l}12 \text { weeks after } \\
\text { Treatment Start }\end{array}$ & 21 & 25.2 & $(10.2,39.1)$ & \\
\hline PFS & $<5$ & Treatment Start & 88 & 17.2 & $(13.3,23.3)$ & \multirow[b]{2}{*}{0.0029} \\
\hline PFS & $\geq 5$ & Treatment Start & 40 & 14 & $(9.8,16.7)$ & \\
\hline PFS & $<5$ & $\begin{array}{l}6 \text { weeks after } \\
\text { Treatment Start }\end{array}$ & 104 & 17.4 & $(14.9,20.5)$ & \multirow[t]{2}{*}{0.037} \\
\hline PFS & $\geq 5$ & $\begin{array}{l}6 \text { weeks after } \\
\text { Treatment Start }\end{array}$ & 27 & 12.1 & $(6.3,15.4)$ & \\
\hline PFS & $<5$ & $\begin{array}{l}12 \text { weeks after } \\
\text { Treatment Start }\end{array}$ & 89 & 18.4 & $(15.4,24.3)$ & \multirow[t]{2}{*}{0.0003} \\
\hline PFS & $\geq 5$ & $\begin{array}{l}12 \text { weeks after } \\
\text { Treatment Start }\end{array}$ & 21 & 5.8 & $(3.4,14.0)$ & \\
\hline OS & $<5$ & Progression & 71 & 33.4 & $(25.2,42.5)$ & \multirow{2}{*}{0.00022} \\
\hline OS & $\geq 5$ & Progression & 31 & 10.2 & $(3.7,17.9)$ & \\
\hline
\end{tabular}

Abbreviations: CI, confidence interval; PFS, progression free survival; OS, overall survival.

\subsection{Univariable Analysis on BMI and Studied Outcomes}

There was no significant difference between patients classified as underweight (BMI $\leq 18.5)$, normal weight (BMI $>18.5$ to $\leq 25)$, overweight (BMI $>25$ to $\leq 30$ ) and obese (BMI > 30), in PFS and OS at therapy start, 6 weeks and 12 weeks. There was no statistically significant difference in OS between BMI categories at time of progression (Figure S2, Table S2). The change in BMI after 6 weeks from therapy start and 12 weeks from therapy start was not associated with PFS ( $p=0.331$ for 6 weeks, $p=0.439$ for 12 weeks) or OS ( $p=0.107$ for 6 weeks, $p=0.174$ for 12 weeks). However, there was an association between BMI change at progression and OS after progression (HR $=0.8371$, $p=0.00336$ ) (Table S3).

No correlation was found between baseline BMI and NLR $(r=-0.145, p=0.104)$. Changes in BMI did not correlate with changes in NLR at 6 weeks of therapy $(r=-0.13$, $p=0.16)$ but significantly correlated at 12 weeks $(r=-0.21, p=0.03)$ (Figure S3).

\subsection{Total Number of Disease Sites, Adverse Drug Events and ECOG Score on NLR}

There was a modest correlation between baseline number of disease sites and NLR at therapy start (Spearman's $\rho=0.272, p=0.002$ ). Throughout the course of treatment, $72 \%$ of patients reported either no or low-grade adverse drug events (grade $0-2$ ), while $28 \%$ of patients experienced high grade $(\geq 3)$ adverse events. There was no difference in NLR at 6 weeks or 12 weeks between patients with high grade versus no or low-grade drug toxicities at 6 weeks $(p=0.28)$ or 12 weeks $(p=0.30)$, and development of grade $3-5$ toxicities did not affect PFS $(p=0.09)$ or OS ( $p=0.8)$. There was no correlation between ECOG score and NLR at therapy start $(r=0.095, p=0.289)$ 


\subsection{Multivariable Analysis on Baseline Patient Characteristics and Survival Outcomes at Treatment Start}

Multivariable cox regression looking at baseline patient characteristics and their relation to OS and PFS are summarized in Table 4 . NLR $\geq 5$ compared to NLR $<5$ at the time of therapy initiation was not associated with increased risk for death or progression after controlling for baseline patient characteristics. Age at diagnosis and gender were not associated with increased risk for progression or for death. African American race did not portend worse OS or PFS when compared to white; however, Asian and other race was associated with worse OS. Baseline number of disease sites at therapy start was an independent factor associated with decreased OS (HR 1.825, 95\% CI 1.328-2.507, $p=0.0002$ ) and decreased PFS (HR 1.473, 95\% CI 1.172-1.851, $p=0.0009$ ). ECOG score of 2-3 compared to 1-2 was strongly associated with decreased OS (HR 4.390, 95\% CI 2.037-9.458, $p=0.0002$ ) and decreased PFS (HR3.435, 95\% CI 1.779-6.634, $p=0.0002$ ). Patients harboring an exon 19 mutation had lower risk for death than patients without the mutation (HR $0.213,95 \%$ CI $0.048-0.948, p=0.042$ ), and patients with G719X mutation had an increased risk for death compared to those without (HR 6.239, 95\% CI 1.026-37.931, $p=0.0047)$. Greater than 15 pack year smoking history was associated with worse OS (HR 4.574, 95\% CI 1.835-11.401, $p=0.001$ ) but not PFS when compared to nonsmokers.

\subsection{Multivariable Analysis of NLR at Treatment Start, 6 Weeks and 12 Weeks of Therapy}

Multivariable analysis of NLR $\geq 5$ compared to $<5$ did not show increased risk for progression at treatment start (HR 1.077, 95\% CI 0.558-1.456 $p=0.801$ ), 6 weeks (HR 1.032, $95 \%$ CI $0.560-1.902, p=0.921$ ) or 12 weeks of therapy (HR 2.075, 95\% CI $0.976-4.412$, $p=0.058$ ). When looking at OS, NLR $\geq 5$ compared to $<5$ did not show an increased risk for death at therapy start (HR 1.935, 95\% CI 0.911-4.108, $p=0.086$ ) or 6 weeks (HR 1.419, 95\% CI 0.645-3.124, $p=0.384$ ); however, NLR $\geq 5$ was associated with increased risk of death at 12 weeks of therapy (HR 3.002, 95\% CI 1.282-7.029, $p=0.011$ ).

Multivariable analysis of NLR using cutoff value of $\geq 3$ compared to $<3$ did not show increased risk of progression at treatment start (HR 1.041, 95\% CI 0.622-1.742, $p=0.879$ ), 6 weeks of therapy (HR $0.911,95 \%$ CI $0.567-1.464, p=0.700$ ) or 12 weeks (HR $0.849,95 \%$ CI $0.489-1.475, p=0.562$ ) nor increased risk of death at 6 weeks (HR 1.683, $95 \%$ CI $0.833-3.398, p=0.147)$. NLR $\geq 3$ was significantly associated with reduced OS at treatment start (HR 2.374, 95\% CI 1.161-4.852, $p=0.018$ ) and at 12 weeks of therapy (HR 2.337, 95\% CI 1.126-4.849, $p=0.023$ )

Multivariable analysis assessing NLR as a continuous variable did not show increased risk of progression at treatment start (HR 0.995, 95\% CI 0.934-1.060, $p=0.871$ ), 6 weeks (HR 1.011, 95\% CI 0.946-1.080, $p=0.746$ ) or 12 weeks (HR 1.123, 95\% CI 0.975-1.293, $p=0.108$ ). When assessing NLR as a continuous variable on OS, there was no statistically significant increased risk of death at treatment start (HR 1.007, 95\% CI 0.943-1.074, $p=0.839$ ) or at 6 weeks (HR 1.043, 95\% CI 0.970-1.121, $p=0.255$ ); however, NLR was found to be significant for OS at the 12-week mark (HR 1.231, 95\% CI 1.063-1.425, $p=0.0054$ ) 
Table 4. Multivariable Cox regression analysis comparing impact of baseline characteristics on overall survival (OS) and progression free survival (PFS) at treatment start.

\begin{tabular}{|c|c|c|c|c|c|c|c|}
\hline \multicolumn{4}{|c|}{ OS } & \multicolumn{4}{|c|}{ PFS } \\
\hline Group & HR $^{1}$ & $95 \%$ CI & $p$ & Group & HR & $95 \%$ CI & $p$ \\
\hline $\begin{array}{l}\text { NLR (pre-treatment) } \\
\quad \geq 5 \text { (vs. <5) }\end{array}$ & 1.935 & $(0.911,4.106)$ & 0.086 & $\begin{array}{l}\text { NLR (pre-treatment) } \\
\quad \geq 5 \text { (vs. <5) }\end{array}$ & 1.077 & $(0.602,1.925)$ & 0.803 \\
\hline $\begin{array}{c}\text { Age } \\
\geq 65 \text { (vs. <65) }\end{array}$ & 1.107 & $(0.574,2.135)$ & 0.7615 & $\begin{array}{c}\text { Age } \\
\geq 65 \text { (vs. <65) }\end{array}$ & 0.902 & $(0.558,1.456)$ & 0.672 \\
\hline $\begin{array}{c}\text { Gender } \\
\text { Male (vs. Female) }\end{array}$ & 0.892 & $(0.410,1.942)$ & 0.773 & $\begin{array}{c}\text { Gender } \\
\text { Male (vs. Female) }\end{array}$ & 16.7 & $(14.5,20.0)$ & 0.052 \\
\hline $\begin{array}{c}\text { Race } \\
\text { Asian (vs. White) } \\
\text { Black (vs. White) } \\
\text { Other (vs. White) }\end{array}$ & $\begin{array}{l}4.274 \\
0.998 \\
5.169\end{array}$ & $\begin{array}{c}(1.399,13.064) \\
(0.424,2.305) \\
(1.191,22.428)\end{array}$ & $\begin{array}{l}0.011 \\
0.978 \\
0.028\end{array}$ & $\begin{array}{c}\text { Race } \\
\text { Asian (vs. White) } \\
\text { Black (vs. White) } \\
\text { Other (vs. White) }\end{array}$ & $\begin{array}{c}0.951 \\
0.72 \\
1.413\end{array}$ & $\begin{array}{l}(0.418,2.167) \\
(0.384,1.348) \\
(0.492,4.061)\end{array}$ & $\begin{array}{l}0.906 \\
0.304 \\
0.521\end{array}$ \\
\hline $\begin{array}{c}\text { Smoking History } \\
\leq 15 \text { pack years (vs. never) } \\
>15 \text { pack years (vs. never) }\end{array}$ & $\begin{array}{l}2.036 \\
4.574\end{array}$ & $\begin{array}{c}(0.884,4.688) \\
(1.835,11.401)\end{array}$ & $\begin{array}{l}0.095 \\
0.001\end{array}$ & $\begin{array}{c}\text { Smoking History } \\
\leq 15 \text { pack years (vs. never) } \\
>15 \text { pack years (vs. never) }\end{array}$ & $\begin{array}{l}1.452 \\
1.754\end{array}$ & $\begin{array}{l}(0.801,2.632) \\
(0.816,3.771)\end{array}$ & $\begin{array}{c}0.219 \\
0.15\end{array}$ \\
\hline $\begin{array}{c}\text { BMI Therapy Start } \\
<18.5 \text { (vs. } 18.5 \text { to } \leq 25) \\
>25 \text { to } \leq 30 \text { (vs. } 18.5 \text { to } \leq 25) \\
>30 \text { (vs. } 18.5 \text { to } \leq 25 \text { ) } \\
\text { Number of Disease Sites } \\
\text { (continuous) }\end{array}$ & $\begin{array}{l}3.85 \\
1.205 \\
0.487\end{array}$ & $\begin{array}{c}(0.575,25.762) \\
(0.563,2.577) \\
(0.177,1.337) \\
(1.328,2.507)\end{array}$ & $\begin{array}{l}0.165 \\
0.631 \\
1.625\end{array}$ & $\begin{array}{c}\text { BMI Therapy Start } \\
<18.5 \text { (vs. } 18.5 \text { to } \leq 25) \\
>25 \text { to } \leq 30 \text { (vs. } 18.5 \text { to } \leq 25) \\
>30 \text { (vs. } 18.5 \text { to } \leq 25) \\
\text { Number of Disease Sites } \\
\text { (continuous) }\end{array}$ & $\begin{array}{l}1.601 \\
1.314 \\
1.155 \\
1.473\end{array}$ & $\begin{array}{l}(0.263,9.751) \\
(0.766,2.253) \\
(0.597,2.234) \\
(1.172,1.851)\end{array}$ & $\begin{array}{c}0.61 \\
0.321 \\
0.669 \\
0.0009\end{array}$ \\
\hline $\begin{array}{c}\text { TKI } \\
\text { Afatinib (vs. osimertinib) }\end{array}$ & 1.123 & $(0.310,4.006)$ & 0.86 & $\begin{array}{c}\text { TKI } \\
\text { Afatinib (vs. osimertinib) }\end{array}$ & 2.267 & $(0.958,5.363)$ & 0.063 \\
\hline $\begin{array}{l}\text { Erlotinib (vs. osimertinib) } \\
\text { TKI Began 1+ Years after } \\
\text { Stage IV Diagnosis }\end{array}$ & 0.529 & $\begin{array}{l}(0.586,4.162) \\
(0.126,2.224)\end{array}$ & $\begin{array}{l}0.372 \\
0.385\end{array}$ & $\begin{array}{l}\text { Erlotinib (vs. osimertinib) } \\
\text { TKI Began 1+ Years after } \\
\text { Stage IV Diagnosis }\end{array}$ & $\begin{array}{l}2.925 \\
0.643\end{array}$ & $\begin{array}{l}(1.501,5.699) \\
(0.238,1.739)\end{array}$ & 0.385 \\
\hline $\begin{array}{l}\text { Received Other Systemic } \\
\text { Treatment before TKI }\end{array}$ & 2.253 & $(0.976,5.203)$ & 0.058 & $\begin{array}{l}\text { Received Other Systemic } \\
\text { Treatment before TKI }\end{array}$ & 1.491 & $(0.815,2.727)$ & 0.195 \\
\hline $\begin{array}{l}\text { ECOG Score } \\
2-3 \text { (vs. 0-1) }\end{array}$ & 4.39 & $(2.037,9.458)$ & 0.0002 & $\begin{array}{l}\text { ECOG Score } \\
\text { (2-3 vs. } 0-1)\end{array}$ & 3.435 & $(1.779,6.634)$ & 0.0002 \\
\hline $\begin{array}{c}\text { Highest Toxicity Grade } \\
3-5 \text { (vs. 0-2) } \\
\text { CNS Metastases }\end{array}$ & $\begin{array}{l}1.475 \\
0.526\end{array}$ & $\begin{array}{l}(0.748,2.909) \\
(0.235,1.177)\end{array}$ & $\begin{array}{l}0.262 \\
0.118\end{array}$ & $\begin{array}{c}\text { Highest Toxicity Grade } \\
3-5 \text { (vs. 0-2) } \\
\text { CNS Metastases }\end{array}$ & $\begin{array}{l}0.837 \\
0.784\end{array}$ & $\begin{array}{l}(0.500,1.400) \\
(0.452,1.358)\end{array}$ & $\begin{array}{l}0.497 \\
0.385\end{array}$ \\
\hline $\begin{array}{c}\text { Baseline Mutation } \\
\text { Exon } 19 \\
\text { L858R } \\
\text { T790M } \\
\text { G719X }\end{array}$ & $\begin{array}{c}0.213 \\
1.44 \\
0.337 \\
6.239\end{array}$ & $\begin{array}{c}(0.048,0.948) \\
(0.331,6.270) \\
(0.101,1.125) \\
(1.026,37.931)\end{array}$ & $\begin{array}{l}0.042 \\
0.627 \\
0.077 \\
0.047\end{array}$ & $\begin{array}{c}\text { Baseline Mutation } \\
\text { Exon } 19 \\
\text { L858R } \\
\text { T790M } \\
\text { G719X }\end{array}$ & $\begin{array}{l}0.339 \\
0.732 \\
0.714 \\
1.014\end{array}$ & $\begin{array}{c}(0.090,1.309) \\
(0.186,2.889) \\
(0.280,1.822) \\
(0.201,5.110\end{array}$ & $\begin{array}{l}0.117 \\
0.656 \\
0.481 \\
0.987\end{array}$ \\
\hline
\end{tabular}

1. HR, Hazard Ratio.

\section{Discussion}

The NLR provides an easily measurable biomarker that warrants further investigation as a predictive biomarker. The biologic reasons why the NLR may be predictive are likely multifactorial. One possibility is that a higher NLR could reflect a more aggressive underlying tumor phenotype. Tumors secrete G-CSF and other soluble mediators that stimulate hematopoiesis [16], and if more aggressive tumors secrete cytokines predominantly skewing towards increased neutrophil production this could result in an elevated NLR [17]. Additionally, there may be a causal relationship where increased numbers of neutrophils compared to lymphocytes further inhibits the anti-tumor response. Activated cytotoxic $\mathrm{CD}^{+} \mathrm{T}$ cells directly kill tumor cells. The activity of these $\mathrm{T}$ cells can be suppressed either by regulatory T cells or myeloid-derived suppressors cells (MDSC). MDSCs are a heterogenous group of cells that can be derived from either a monocytic or neutrophilic lineage [18]. Neutrophils can secrete soluble factors including reactive oxygen species (ROS) and arginase 1 to inhibit T cells [19]. Tumor associated neutrophils (TAN) are another population of cells that can be either pro- or anti-tumorigenic. Neutrophil extracellular traps (NETs) have been associated with increasing the metastatic potential of tumor cells 
and neutrophils can also secrete factors such as VEGF which promote tumor angiogenesis [20-22]. Future work is needed to further understand the biologic underpinnings of the clinical correlation between NLR and prognosis and identify novel strategies to target the EGFR tumor microenvironment in patients with high NLR.

One question is whether a change in the NLR during treatment is related to upcoming progression. There was no significant change in the NLR from the start of treatment to 6 weeks prior to progression (mean $=-0.9702, \mathrm{SD}=5.6207, n=86, p=0.1786$ ), or to the time of progression (mean $=0.4406, \mathrm{SD}=7.3476, n=96, p=0.5582$ ). When comparing NLR at progression and NLR at start of therapy amongst patients who progressed $(n=96)$, NLR on average increased; however, this finding was not statistically significant (mean 0.441 , SD 7.348, $p=0.558$ ). There was also no significant difference in PFS and OS for patients whose NLR decreased at 6 and 12 weeks compared to those whose NLR increased at 6 and 12 weeks, which led us to conclude that changes in NLR do not have any utility in predicting progression or prognosis.

Another factor that has been shown to affect prognosis in lung cancer is BMI. No significant difference in OS was noted between the different BMI groups at therapy start, 6 weeks, 12 weeks and progression. After adjusting for covariates, BMI was not an independent predictive factor for OS and PFS when comparing patients classified as underweight, overweight and obese to patients with normal BMI. A change in BMI after 6 weeks or 12 weeks from therapy start was not correlated with PFS ( $p=0.331$ for 6 weeks, $p=0.439$ for 12 weeks) or OS ( $p=0.107$ for 6 weeks, $p=0.174$ for 12 weeks); however, there was a correlation noted between BMI change (from therapy start to progression) and OS after progression ( $\mathrm{HR}=0.8371, p=0.00336$. One potential explanation for these findings is that cancer-mediated cachexia is a slow process that becomes evident over longer periods of time as opposed to within the first few months of initiating therapy.

Prognostic stratification of patients by NLR has therapeutic implications. Combining TKI therapy with chemotherapy in patients with an extensive disease burden may offer improvements in PFS and OS [23]. FLAURA2 is a phase III randomized trial underway to determine if combining osimertinib with carboplatin/pemetrexed improves PFS and OS (ClinicalTrials.gov accessed on 22 March 2021 Identifier NCT04035486). Antiangiogenic agents are also being pursued (ClinicalTrials.gov accessed on 22 March 2021 Identifier NCT04181060) with a recent publication suggesting bevacizumab and osimertinib led to a favorable 1-year PFS [24] and other trials that are ongoing as well are trials adding the antiangiogenic agent ramucirumab (ClinicalTrials.gov accessed on 22 March 2021 Identifier NCT03909334). Studying the NLR in patients treated with angiogenic agents would also be useful in light of recent FDA approval of ramucirumab with erlotinib in front-line treatment of metastatic NSCLC with EGFR exon 19 deletions or exon 21 (L858R) mutations [25]. Using the NLR at 12 weeks of therapy to identify patients with a poor prognosis may help select the population most likely to benefit from early intensification of treatment. Additionally, as novel therapeutics targeting the suppressive effects of neutrophils are developed, the NLR could be used as a predictive factor to stratify patients who may be most likely to benefit from these strategies in combination with a TKI.

Our study does have several limitations. It is a retrospective study, and information was obtained from standard clinical documentation. Additionally, we used clinical notes and change in therapy to determine progression instead of RECIST criteria however as many patients are kept on TKIs with minimal progression and with the application of local therapies, we feel this is a minor weakness. Some patients did not have the NLR measurements available at all timepoints examined or had NLR examined from a hospital stay. We feel this is a minor weakness as we did not see a correlation between NLR and ECOG performance status, which could reflect underlying reasons for hospital admission We also were not able to analyze effects of co-mutations such as those in $R b$ and p53 or circulating tumor DNA clearance which have been shown to portend poor prognosis $[26,27]$. Furthermore, since toxicity data was taken from the treating physician's medical record notes it may not be as complete as toxicity data which is prospectively documented for 
patients enrolled in a clinical trial. Finally, during the time course of our study the standard of care TKI shifted to the third-generation drug, osimertinib. While over $25 \%$ of our cohort was treated with osimertinib as their initial TKI, our study also included patients treated with older generation TKIs and some who had initiated treatment with an earlier generation TKI while awaiting approval for a newer generation.

Previous studies examining the effect of NLR on an EGFR-mutated population have largely been done in Asian populations [13]. One strength of our study is that data is pooled from two institutions in Chicago with a racially diverse patient population, and the patient race distribution was reflective of the study location. Our study cohort was $22 \%$ African American, which is important given the paucity of data that exists which addresses racial disparities among patients with EGFR mutated lung cancer. Cheng et al. (2020) reported inferior survival among black patients with NSCLC with EGFR mutations as compared to non-black patients, with 2-year survival rates being $33 \%$ versus $61 \%$, respectively $(p=0.001)$ [28]. Shorter survival was also observed for those patients treated with targeted EGFR therapy. Earlier studies have hypothesized that the survival discrepancy may be more a consequence of socioeconomic disparities as opposed to genetic factors [29-31]. Most of these studies were obtained before targeted therapy became standard of care. We did not find a statistically significant difference in PFS and OS between African American and non-African American patients but, nevertheless, further investigation on racial disparities and survival outcomes in this new TKI era are warranted.

Prior studies have looked solely at pretreatment NLR and have found that pretreatment NLR is an independent predictor of PFS and OS [9,32]. We did not observe NLR at the pretreatment stage (i.e., time of therapy initiation) to be an independent predictive factor as prior studies have reported, possibly due to our limited sample size and the strong association between baseline number of disease sites with OS and PFS, and ECOG score with OS and PFS. Our work nevertheless highlights the value of serial NLR measurements collected at multiple time points throughout TKI therapy and not just at the pre-treatment stage, as NLR is a dynamic value that is expected to fluctuate throughout disease course and treatment. We validate previous studies demonstrating that elevated NLR is a poor prognostic sign and further extend this finding to include patients treated with the third generation TKI osimertinib.

\section{Conclusions}

The results of our study supporting NLR as a predictive biomarker for patients with advanced stage EGFR mutated NSCLC on TKI therapy is concordant with what has been previously described in the literature. We highlight the predictive value of NLR on OS particularly at the 12-week mark after initiation of therapy. Based on these results, increased frequency of monitoring and potential therapeutic escalation may be beneficial in patients with elevated NLR $\geq 5$, particularly after receiving 12 weeks of TKI therapy.

Supplementary Materials: The following are available online at https:/ /www.mdpi.com/2072-6 694/13/6/1426/s1, Figure S1. Progression free survival probability for NLR < 3 (red) compared to NLR $\geq 3$ (blue) at (a) treatment start, (b) 6 weeks of therapy and (c) 12 weeks of therapy, Figure S2: Progression free survival probability for BMI $\leq 18.5$ (red), $>18.5$ to $\leq 25$ (green), $>25$ to $\leq 30$ (blue) and $>30$ (purple) at (a) treatment start, (b) 6 weeks of therapy and (c) 12 weeks of therapy. Overall survival probability for $\mathrm{BMI} \leq 18.5,>18.5$ to $\leq 25,>25$ to $\leq 30$ and $>30$ at (d) treatment start, (e) 6 weeks of therapy and (f) 12 weeks of therapy, Figure S3. Correlation between change in BMI and change in NLR between treatment initiation and 12 weeks of therapy, Table S1. Progression free survival (PFS) and overall survival (OS) for patients with NLR $\geq 3$ and $<3$ at therapy start, 6 weeks and 12 weeks after beginning treatment, Table S2: Progression free survival (PFS) and overall survival (OS) for patients with $\mathrm{BMI} \leq 18.5,>18.5$ to $\leq 25,>25$ to $\leq 30$ and $>30$ at treatment start, 6 weeks after therapy and 12 weeks after therapy and OS at progression for patients with BMI $\leq 18.5,>18.5$ to $\leq 25,>25$ to $\leq 30$ and $>30$, Table S3: Change in BMI on OS and PFS.

Author Contributions: N.K.Y. and S.J.R. contributed to the methodology, data curation, writingoriginal draft preparation and writing-review and editing, investigation, visualization and formal 
analysis of this project. C.M.B. contributed to conceptualization, methodology, validation, investigation, data curation, writing-original draft preparation, writing-review and editing, formal analysis and supervision. E.M.R. contributed to formal analysis, data curation, software, visualization and writing-original draft preparation and writing-review and editing. B.A.G. contributed to the conceptualization, methodology, data curation, investigation and formal analysis of this project. I.T. and J.A.B. contributed to conceptualization, methodology, validation, investigation, resources, data curation and supervision. M.B. and P.D.B. contributed to conceptualization, methodology, validation, investigation, resources, data curation and supervision. M.J.F. contributed to conceptualization, methodology, validation, investigation, data curation, writing-original draft preparation, writingreview and editing, supervision and formal analysis of this project. All authors have read and agreed to the published version of the manuscript.

Funding: This research received no external funding.

Institutional Review Board Statement: The study was conducted according to the guidelines of the Declaration of Helsinki and approved by the Institutional Review Board of Rush University (IRB \#13030801 originally approved on 18 April 2013 with latest continuing review on 5 December 2020) and the Institutional Review Board of The University of Chicago (IRB \#20-0366 on 6 August 2020).

Informed Consent Statement: Patient consent was waived in our retrospective study given that it posed minimal risk and no direct patient contact was made.

Data Availability Statement: The data that support the findings of this study are available from the corresponding author, M.J.F., upon reasonable request. Data are not made publicly available due to privacy or ethical restrictions.

Conflicts of Interest: N.K.Y., S.J.R., E.M.R. and M.B. have no disclosures. C.M.B. participated in an advisory board with AbbVie, Astrazeneca, Genetech, Pfizer, received an honorarium from OncLive, and has a research grant from Bristol-Myers Squibb. B.A.G. participated in a Speaker's Bureau with Astrazeneca. I.T. has received travel reimbursement from Luminex. J.A.B. consulted for and participated in Speaker's Bureaus for Luminex and MilliporeSigma, and has research support from Pfizer, Biodesiz, Abbott Diagnostics, Epigenomics. P.D.B. has received honoraria for advisory board participation from AstraZeneca, Biodesix, Helsinn, Merck, Pfizer, and Spectrum. M.J.F. has participated in Speakers bureaus for Novartis, Genetech, and Merck, consulted for Genentech, Abbvie, Guardant Health, AstraZeneca, Rakuten, and has received research support from Biodesix, Pfizer/EMD Serono, Astra Zeneca, Jounce, CytomX Therapeutics, Merck, Novartis, Rakuten.

\section{References}

1. Siegel, R.L.; Miller, K.D.; Jemal, A. Cancer statistics, 2020. CA Cancer J. Clin. 2020, 70, 7-30. [CrossRef]

2. Aguiar-Bujanda, D.; Dueñas-Comino, A.; Saura-Grau, S.; Ros-Sanjuan, L.; Blanco-Sanchez, M.J.; Hernandez-Sosa, M.; Santiago, M.M.-D.; Galvan-Ruiz, S.; Lorenzo-Barreto, J.E.; Vargas-Prado, A.M.; et al. Neutrophil to Lymphocyte Ratio as a Prognostic Factor in European Patients with Epidermal Growth Factor Receptor-Mutant Non-Small Cell Lung Cancer Treated with Tyrosine Kinase Inhibitors. Oncol. Res. Treat. 2018, 41, 755-761. [CrossRef]

3. Ramalingam, S.S.; Vansteenkiste, J.; Planchard, D.; Cho, B.C.; Gray, J.E.; Ohe, Y.; Zhou, C.; Reungwetwattana, T.; Cheng, Y.; Chewaskulyong, B.; et al. Overall Survival with Osimertinib in Untreated, EGFR-Mutated Advanced NSCLC. N. Engl. J. Med. 2020, 382, 41-50. [CrossRef]

4. Fang, W.; Huang, Y.; Hong, S.; Zhang, Z.; Wang, M.; Gan, J.; Wang, W.; Guo, H.; Wang, K.; Zhang, L. EGFR exon 20 insertion mutations and response to osimertinib in non-small-cell lung cancer. BMC Cancer 2019, 19, 595. [CrossRef]

5. Derman, B.A.; Macklis, J.N.; Azeem, M.S.; Sayidine, S.; Basu, S.; Batus, M.; Esmail, F.; Borgia, J.A.; Bonomi, P.; Fidler, M.J. Relationships between longitudinal neutrophil to lymphocyte ratios, body weight changes, and overall survival in patients with non-small cell lung cancer. BMC Cancer 2017, 17, 141. [CrossRef] [PubMed]

6. Forget, P.; Khalifa, C.; Defour, J.P.; Latinne, D.; Van Pel, M.C.; De Kock, M. What is the normal value of the neutrophil-tolymphocyte ratio? BMC Res. Notes 2017, 10, 12. [CrossRef] [PubMed]

7. Proctor, M.J.; McMillan, D.C.; Morrison, D.S.; Fletcher, C.D.; Horgan, P.G.; Clarke, S.J. A derived neutrophil to lymphocyte ratio predicts survival in patients with cancer. Br. J. Cancer 2012, 107, 695-699. [CrossRef]

8. Zhang, Y.; Feng, Y.-C.; Zhu, H.-G.; Xiong, T.-C.; Hou, Y.-S.; Song, J.; Jiang, W.; Zhu, C.-J. The peripheral blood neutrophilto-lymphocyte ratio is a prognostic predictor for survival of EGFR-mutant nonsmall cell lung cancer patients treated with EGFR-TKIs. Medicine 2018, 97, e11648. [CrossRef]

9. Wang, Z.; Zhan, P.; Lv, Y.; Shen, K.; Wei, Y.; Liu, H.; Song, Y. Prognostic role of pretreatment neutrophil-to-lymphocyte ratio in non-small cell lung cancer patients treated with systemic therapy: A meta-analysis. Transl. Lung Cancer Res. 2019, 8, 214-226. [CrossRef] [PubMed] 
10. Minami, S.; Ogata, Y.; Ihara, S.; Yamamoto, S.; Komuta, K. Neutrophil-to-Lymphocyte Ratio Predicts Overall Survival of Advanced Non-Small Cell Lung Cancer Harboring Mutant Epidermal Growth Factor Receptor. World J. Oncol. 2017, 8, 180-187. [CrossRef]

11. Phan, T.T.; Ho, T.T.; Nguyen, H.T.; Nguyen, H.T.; Tran, T.B.; Nguyen, S.T. The prognostic impact of neutrophil to lymphocyte ratio in advanced non-small cell lung cancer patients treated with EGFR TKI. Int. J. Gen. Med. 2018, 11, 423-430. [CrossRef]

12. Deng, H.Y.; Hou, L.; Zha, P.; Huang, K.L.; Peng, L. Sarcopenia is an independent unfavorable prognostic factor of non-small cell lung cancer after surgical resection: A comprehensive systematic review and meta-analysis. Eur. J. Surg. Oncol. 2019, 45, 728-735. [CrossRef]

13. Ono, T.; Igawa, S.; Kurahayashi, S.; Okuma, Y.; Sugimoto, A.; Kusuhara, S.; Ozawa, T.; Fukui, T.; Sasaki, J.; Mitsufuji, H.; et al. Impact of neutrophil-to-lymphocyte ratio in patients with EGFR-mutant NSCLC treated with tyrosine kinase inhibitors. Investig. New Drugs 2020. [CrossRef]

14. Meriggi, F.; Codignola, C.; Beretta, G.D.; Ceresoli, G.L.; Caprioli, A.; Scartozzi, M.; Fraccon, A.P.; Prochilo, T.; Ogliosi, C.; Zaniboni, A. Significance of neutrophil-to-lymphocyte ratio in Western advanced EGFR-mutated non-small cell lung cancer receiving a targeted therapy. Tumori J. 2017, 103, 443-448. [CrossRef] [PubMed]

15. Mandaliya, H.; Jones, M.; Oldmeadow, C.; Nordman, I.I. Prognostic biomarkers in stage IV non-small cell lung cancer (NSCLC): Neutrophil to lymphocyte ratio (NLR), lymphocyte to monocyte ratio (LMR), platelet to lymphocyte ratio (PLR) and advanced lung cancer inflammation index (ALI). Transl. Lung Cancer Res. 2019, 8, 886-894. [CrossRef] [PubMed]

16. Wislez, M.; Fleury-Feith, J.; Rabbe, N.; Moreau, J.; Cesari, D.; Milleron, B.; Mayaud, C.; Antoine, M.; Soler, P.; Cadranel, J. Tumorderived granulocyte-macrophage colony-stimulating factor and granulocyte colony-stimulating factor prolong the survival of neutrophils infiltrating bronchoalveolar subtype pulmonary adenocarcinoma. Am. J. Pathol. 2001, 159, 1423-1433. [CrossRef]

17. Tavakkoli, M.; Wilkins, C.R.; Mones, J.V.; Mauro, M.J. A Novel Paradigm Between Leukocytosis, G-CSF Secretion, Neutrophil-toLymphocyte Ratio, Myeloid-Derived Suppressor Cells, and Prognosis in Non-small Cell Lung Cancer. Front Oncol. 2019,9 , 295. [CrossRef]

18. Tcyganov, E.; Mastio, J.; Chen, E.; Gabrilovich, D.I. Plasticity of myeloid-derived suppressor cells in cancer. Curr. Opin. Immunol. 2018, 51, 76-82. [CrossRef]

19. Singel, K.L.; Segal, B.H. Neutrophils in the tumor microenvironment: Trying to heal the wound that cannot heal. Immunol. Rev. 2016, 273, 329-343. [CrossRef]

20. Yang, L.-Y.; Luo, Q.; Lu, L.; Zhu, W.-W.; Sun, H.-T.; Wei, R.; Lin, Z.-F.; Wang, X.-Y.; Wang, C.-Q.; Lu, M.; et al. Increased neutrophil extracellular traps promote metastasis potential of hepatocellular carcinoma via provoking tumorous inflammatory response. J. Hematol. Oncol. 2020, 13, 3. [CrossRef]

21. Hurt, B.; Schulick, R.; Edil, B.; El Kasmi, K.C.; Barnett, C., Jr. Cancer-promoting mechanisms of tumor-associated neutrophils. Am. J. Surg. 2017, 214, 938-944. [CrossRef]

22. Jaillon, S.; Ponzetta, A.; Di Mitri, D.; Santoni, A.; Bonecchi, R.; Mantovani, A. Neutrophil diversity and plasticity in tumour progression and therapy [published online ahead of print, $2020 \mathrm{Jul}$ 21]. Nat. Rev. Cancer 2020. [CrossRef]

23. Rebuzzi, S.E.; Alfieri, R.; La Monica, S.; Minari, R.; Petronini, P.G.; Tiseo, M. Combination of EGFR-TKIs and chemotherapy in advanced EGFR mutated NSCLC: Review of the literature and future perspectives. Crit. Rev. Oncol. Hematol. 2020, 146, 102820. [CrossRef]

24. Yu, H.A.; Schoenfeld, A.J.; Makhnin, A.; Kim, R.; Rizvi, H.; Tsui, D.; Falcon, C.; Houck-Loomis, B.; Meng, F.; Yang, J.L.; et al. Effect of Osimertinib and Bevacizumab on Progression-Free Survival for Patients With Metastatic EGFR-Mutant Lung Cancers: A Phase 1/2 Single-Group Open-Label Trial. JAMA Oncol. 2020, 6, 1048-1054. [CrossRef]

25. Nakagawa, K.; Garon, E.B.; Seto, T.; Nishio, M.; Ponce Aix, S.; Paz-Ares, L.; Chiu, C.H.; Park, K.; Novello, S.; Nadal, E.; et al. Ramucirumab plus erlotinib in patients with untreated, EGFR-mutated, advanced non-small-cell lung cancer (RELAY): A randomised, double-blind, placebo-controlled, phase 3 trial. Lancet Oncol. 2019, 1655-1669. [CrossRef]

26. Marcoux, N.; Gettinger, S.N.; O’Kane, G.; Arbour, K.C.; Neal, J.W.; Husain, H.; Evans, T.L.; Brahmer, J.R.; Muzikansky, A.; Bonomi, P.D.; et al. EGFR-Mutant Adenocarcinomas That Transform to Small-Cell Lung Cancer and Other Neuroendocrine Carcinomas: Clinical Outcomes. J. Clin. Oncol. 2019, 278-285. [CrossRef]

27. Zhou, C.; Imamura, F.; Cheng, Y.; Okamoto, I.; Cho, B.C.; Lin, M.C.; Majem, M.; Gautschi, O.; Gray, J.E.; Boyer, M.J.; et al. Early clearance of plasma EGFR mutations as a predictor of response to Osimertinib and comparator EGFR-TKIs in the FLAURA trial. J. Clin. Oncol. 2019, 37 (Suppl. 15), 9020. [CrossRef]

28. Cheng, H.; Hosgood, H.D.; Deng, L.; Ye, K.; Su, C.; Sharma, J.; Yang, Y.; Halmos, B.; Perez-Soler, R. Survival Disparities in Black Patients With EGFR-mutated Non-small-cell Lung Cancer. Clin. Lung Cancer 2020, 177-185. [CrossRef] [PubMed]

29. Leduc, N.; Atallah, V.; Agossou, M.; Vinh-Hung, V.; Orre, M.; Sargos, P.; Molinie, V. Lung Adenocarcinoma Survival in EGFRMutated African-Caribbean Patients: A Multicenter Study in the French West Indies. Target Oncol. 2017, 689-693. [CrossRef] [PubMed] 
30. Jones, C.C.; Mercaldo, S.F.; Blume, J.D.; Wenzlaff, A.S.; Schwartz, A.G.; Chen, H.; Deppen, S.A.; Bush, W.S.; Crawford, D.C.; Chanock, S.J.; et al. Racial Disparities in Lung Cancer Survival: The Contribution of Stage, Treatment, and Ancestry. J. Thorac. Oncol. 2018, 1464-1473. [CrossRef]

31. Ellis, L.; Canchola, A.J.; Spiegel, D.; Ladabaum, U.; Haile, R.; Gomez, S.L. Racial and Ethnic Disparities in Cancer Survival: The Contribution of Tumor, Sociodemographic, Institutional, and Neighborhood Characteristics. J. Clin. Oncol. 2018, 36, 25-33. [CrossRef]

32. Tang, M.; Gao, X.; Sun, H.; Tian, S.; Dong, J.; Liu, Z.; Liu, W. Neutrophil-Lymphocyte Ratio as a Prognostic Parameter in NSCLC Patients Receiving EGFR-TKIs: A Systematic Review and Meta-Analysis. J. Oncol. 2021, 2021, 6688346. [CrossRef] [PubMed] 